\title{
Children's and Adolescents' Pain, Discomfort, Fear, Cooperation, and Parental Presence During Dental Extractions
}

\author{
Larisa Krekmanova* and Agneta Robertson \\ Department of Pediatric Dentistry, Institute of Odontology at the Sahlgrenska Academy, University of Gothenburg, Sweden
}

*Corresponding author: Larisa Krekmanova, Department of Pediatric Dentistry, Institute of Odontology at the Sahlgrenska Academy, University of Gothenburg, Gothenburg, Sweden

\begin{abstract}
Introduction: Children's reports of experiences from dental invasive procedures are scarce. The aim was to renew and actualize the understanding of children's experiences of pain, discomfort, fear, and cooperation during the dental extraction procedures. A further aim was to study the frequency of parental presence during dental extractions.

Methods: The study was based on a sample of children and adolescents aged 3-19 years in the Region Västra Götaland (RVG) and Region Örebro County (ROC). It was a 5-year cohort study of an accelerated longitudinal design, named BITA (Barn I TAndvården = Children in dental care). Data on children's pain, discomfort, fear, cooperation, and parental presence were assessed and compiled.

Results: 2363 out of 3134 children in the four age cohorts became eligible for inclusion. The cohorts included Cohort 1 (3-7 years old; $n=695$ children), Cohort 2 ( $7-11$ years old; $n=642$ children), Cohort 3 (11-15 years old; $n=574$ children), and Cohort 4 (15-19 years old; $n=452$ children). There were 1215 girls (51\%) and 1148 boys (49\%). Extractions were assessed as painful by $62 \%$. Discomfort was reported by $33 \%$. Dental fear was reported by $47 \%$. During painful extractions, no fear was reported by $38 \%$, while in painless extractions, no fear was reported by $63 \%$. Dental fear was most common in the age group 11-15 years. Full treatment acceptance was recorded in $73.6 \%$ during the extractions. In extractions reported to cause pain, $61 \%$ cooperated well. Most of the patients (77\%) showed full treatment acceptance with parental presence in the dental room. The corresponding figure was $55 \%$ for the parent not being present.
\end{abstract}

Conclusion: Dental extractions continue to constitute one of the most complex and challenging treatment situations for the young patient. The oral injection was most frequently reported to cause pain, discomfort, and fear during dental extractions. Dentists should make efforts to prevent pain and discomfort, as well as utilize parental support for the child's sense of security during dental extractions.

Keywords: Paediatric; pain management; pain assessment; clinical procedures

Abbreviations: RVG: Region Västra Götaland, ROC: Region Örebro County; BITA: Barn I T Andvården; DFA: Dental Fear and Anxiety; BMP: Behavior Management Problems

\section{Introduction}

Invasive dental treatments such as anesthesia and extractions have shown to stress children and adolescents the most [1,2]. In a study performed on 368 Swedish children and adolescents (819 years), the prevailing reported painful dental procedures were injection, tooth extraction, drilling and performing a filling [1]. Negative treatment experiences may trigger a vicious cycle, i.e., leading to avoidance of dental appointments and hindering more positive experiences to take place [3-5]. Furthermore, avoiding dental appointments may affect the oral health [3-5] jeopardizing the well-being of young patients with long-term consequences [3-5]. Less traumatizing methods for invasive dental techniques during injection and extraction are available, although to date, studies report that the usage of pain preventive interventions 
such as topical anesthesia, local anesthesia, or analgesics are still substandard [6-8]. The routines applied by Swedish dentists have shown that 'about 35\% were more indifferent to their patient's experiences of pain and psychological management' [7]. Apart from pain experiences, invasive and/or noninvasive dental procedures may cause children discomfort. Although there is no universally prevailing definition, the phenomenon might be explained as psychological or bodily distress or annoyance, i.e., anything that disturbs the well-being [9]. Children's experience of discomfort in dentistry has previously not been given much attention. Invasive procedures also test dentists' technical skills with a need to be knowledgeable on children's developmental levels, contemporary psychology, and pedagogical interactions. Furthermore, dentists may face difficulties in the interaction with the child/parent dyad [10].

Parental attitudes and behavior have been suggested to either facilitate or undermine the child's cooperation and thus affect the treatment outcome [11-14]. It could be hypothesized that some dentists consider parents a hindrance, therefore not inviting them into the dental room. Seen from the young patients' standpoint, such an approach may be controversial as parental absence may diminish the perception of security, predisposing to fear, pain, and discomfort experiences [14]. In Sweden, a prevailing tradition and the Dental Act have allowed parents to support their children in medical and dental settings [15]. The practice has thus become a norm in the pediatric dental community, despite alternative behavior management techniques [16]. At present, there is insufficient knowledge in the area of children's and adolescents' experience of invasive procedures, and dentists' attitudes and praxis towards these. Furthermore, there is a lack of knowledge on how general dentists understand the parental interaction and how frequently they make use of parental help during extractions. The aim was to renew and actualize the understanding of children's experiences of pain, discomfort, fear, and cooperation during dental extraction procedures. A further aim was to study the frequency of parental presence during dental extractions.

\section{Patients and Methods}

\section{Patients}

This study was based on a representative sample of children and adolescents aged 3-19 years in the Region Västra Götaland (RVG) and Region Örebro County (ROC). The project was a 5-year cohort study of an accelerated longitudinal design, named BITA (Barn I TAndvården = Children in dental care). It concerned different aspects of children in the dental situation when attending regular general dentistry. Four age cohorts with children aged 3, 7, 11 and 15 years, from seven Public Dental Service clinics in RVG and five in ROC, were invited to participate and then followed over a 5-year period in conjunction with regular dental visits. The clinics were selected to reflect the respective populations and to cover urban and rural areas. A total of 3134 children were considered for the study.

\section{Procedure}

\section{Clinical registrations}

The children's ordinary dentists registered all performed dental treatments after each session. For this study, extraction treatments were selected, and assessments and self-ratings were made and registered.

\section{Self-reported pain}

Each child made a self-assessment of pain experienced after the extraction. The child was asked the question: Was anything painful? Yes or no? If the child answered yes, the pain intensity was measured using a visual analog scale (VAS) consisting of 11 points running from $0=$ no pain, to $10=$ worst pain possible. Parallel to the scale, six faces express different levels of pain/distress where young children point out the face matching their level of pain. Furthermore, the child was asked the question: What was painful?

\section{Self-reported discomfort}

The child was asked the question: Did anything cause discomfort? Yes or no? If the child answered yes, the discomfort was measured using a visual rating scale (VRS) consisting of 11 points running from $0=$ no discomfort, to $10=$ worst discomfort possible. Parallel to the scale, six faces express different levels of discomfort where young children point out the face matching their level of discomfort. Furthermore, the child was asked the question: What caused the discomfort?

\section{Self-reported fear}

Children assessed their fear at the dental treatment by answering the question, how did you feel today?

The alternative answers were Not afraid at all $=0$ or Afraid, on a scale graded 1-4.

\section{Assessment of the child's cooperation}

The child's cooperation was graded by the treating dental personnel according to the scale by Rud and Kisling, rated 3 to 0 , where 3 = full acceptance to treatment; 2 = indifferent acceptance; $1=$ reluctant acceptance; and $0=$ non-acceptance.

\section{Parental presence}

Parental presence in the dental room was documented.

\section{Ethics}

The application for ethical review (No. 286-07) was approved.

\section{Statistical methods}

In addition to descriptive statistics in terms of frequency distributions, means, and standard deviations, differences between 
genders and between cohorts were analyzed using Chi-square tests. The statistical analyses were performed in IBM SPSS Statistics for Windows, Version 23.0 (IBM Corp., Armonk, NY, USA ) and p values below 0.05 represented statistical significance.

\section{Results}

3134 children were invited to participate in the study; 771 declined participation, whereby 2363 children in the four age cohorts became eligible for inclusion. These four age cohorts included Cohort 1 (born 2005; from 3-7 years old during the study period; $\mathrm{n}=695$ children, 3906 visits), Cohort 2 (born 2001; 7-11 years old; $n=642$ children, 4588 visits), Cohort 3 (born 1997; 11-15 years old; $n=574$ children, 4085 visits), and Cohort 4 (born 1993; 15-19 years old; $n=452$ children, 3315 visits). The distribution between the genders was 1215 girls (51\%) and 1148 boys (49\%).

\section{Pain and discomfort during extractions}

Extractions were assessed as painful by $62 \%$ (Table 1). The reasons were the extraction itself (16\%), the injection (72\%) or the combination of injection and extraction procedures (6\%) (Table 2). Discomfort was reported in 33\% of the extractions (Table 1). The reasons for discomfort pointed to the extraction per se (37\%), followed by injection (26\%) (Table 2). No statistically significant difference between the genders or cohorts was found regarding the experience of pain. There was a statistically significant difference between cohorts, with the lowest proportion of discomfort stated by the oldest group $(30 \%, 34 \%, 39 \%, 15 \%$; $p=0.026)$ (Table 1). Primary $(n=323)$ and permanent $(n=102)$ tooth extractions were assessed as painful in equal proportions (63\% and 61\%), while discomfort was slightly less often reported for primary teeth, compared to permanent tooth extractions (32\% and 37\%). 11 tooth extractions lacked complete documentation. Topical anesthesia was applied in 35\%, with no statistically significant difference between application and no application regarding the reported experience of pain and/or discomfort. Conscious sedation drugs were used in $6 \%$.

Table 1: Extraction appointments ( $\mathrm{n}=436$ extractions) and the young patients', reports of pain and discomfort in adjunction to the treatment, related to gender and age-cohorts.

\begin{tabular}{|c|c|c|c|c|c|c|c|c|c|c|}
\hline & Pain & No pain & Total & $\mathbf{p}$ & Missing & $\begin{array}{c}\text { Dis- } \\
\text { comfort }\end{array}$ & No discomfort & Total & $\mathbf{p}$ & Missing \\
\hline Girls & $146(63 \%)$ & $85(37 \%)$ & 231 & & & $83(37 \%)$ & $141(63 \%)$ & 224 & & \\
\hline Boys & $124(61 \%)$ & 78 (39\%) & 202 & & & 57 (29\%) & 139 (71\%) & 196 & & \\
\hline Total & $270(62 \%)$ & 163 (38\%) & 433 & 0.697 & & $140(33 \%)$ & $280(67 \%)$ & 420 & 0.084 & \\
\hline \multicolumn{11}{|c|}{ Years } \\
\hline 7-Mar & $23(59 \%)$ & $16(41 \%)$ & 39 & & & $11(30 \%)$ & $26(70 \%)$ & 37 & & 2 \\
\hline 11-Jul & $111(66 \%)$ & $56(34 \%)$ & 167 & & 1 & $55(34 \%)$ & $109(66 \%)$ & 164 & & 3 \\
\hline 15-Nov & $111(61 \%)$ & 70 (39\%) & 181 & & 2 & 67 (39\%) & $106(61 \%)$ & 173 & & 10 \\
\hline $15-19$ & 25 (54\%) & $21(46 \%)$ & 46 & & & 7 (15\%) & $39(85 \%)$ & 46 & & \\
\hline Total & $270(62 \%)$ & $163(38 \%)$ & 433 & 0.435 & & $140(33 \%)$ & $280(67 \%)$ & 420 & 0.026 & \\
\hline
\end{tabular}

Chi-2 test, P probability value

Table 2: Children's reported reasons for pain $(n=270)$ and discomfort $(n=140)$ in connection to the extraction procedures.

\begin{tabular}{|c|c|c|c|c|}
\hline & Pain n & (\%) & Discomfort n & (\%) \\
\hline Extraction & 43 & $(16)$ & 49 & $(35)$ \\
\hline Injection & 193 & $(72)$ & 37 & $(26)$ \\
\hline Extraction and & & & & \\
\hline injection & 17 & $(6)$ & 16 & $(11)$ \\
\hline Sound & & & 12 & $(9)$ \\
\hline Numbness & & & 11 & $(8)$ \\
\hline Taste & & & 2 & $(6)$ \\
\hline Negative expectation & 17 & $(6)$ & 5 & $(1)$ \\
\hline Blood & 270 & & 140 & $(4)$ \\
\hline Unknown & & & & \\
\hline Total & & & & \\
\hline
\end{tabular}




\section{Dental fear and pain during extractions}

Children's self-assessment of dental fear resulted in $47 \%$ reporting no dental fear in conjunction with the extractions. During painful extractions, no fear was reported by $38 \%$, while in painless extractions, no fear was reported by $63 \%$ (Table 3). Dental fear was most common in the age group 11-15 years, followed by the age group 7-11 years (Table 3). No statistically significant difference between the genders or cohorts was found regarding the experience of pain. There was a statistically significant difference between cohorts, with the lowest proportion of discomfort stated by the oldest group (30\%, 34\%, 39\%, 15\%; $\mathrm{p}=0.026$ ) (Table 1). Primary $(n=323)$ and permanent $(n=102)$ tooth extractions were assessed as painful in equal proportions (63\% and 61\%), while discomfort was slightly less often reported for primary teeth, compared to permanent tooth extractions (32\% and 37\%). 11 tooth extractions lacked complete documentation. Topical anesthesia was applied in $35 \%$, with no statistically significant difference between application and no application regarding the reported experience of pain and/ or discomfort. Conscious sedation drugs were used in $6 \%$.

Table 3: Young patients' (age cohorts) self-rated dental fear and pain experiences. The subjective assessment of pain=Yes or No was given after the extraction procedure (missing data $\mathrm{n}=14)$. Dental fear, as rated on a scale 0 to $4,0=$ no fear, fear $=1-4$, (missing data $\mathrm{n}=12$ ).

\begin{tabular}{|c|c|c|c|c|c|c|c|c|c|}
\hline Ages Yrs & 7-Mar & 11-Jul & 15-Nov & 15-19 & Total & Pain & No pain & Total \\
\hline \multicolumn{7}{|c|}{ Fear Scale patient } \\
\hline 0 & $19(54 \%)$ & $79(48 \%)$ & $77(43 \%)$ & $26(57 \%)$ & $201(47 \%)$ & $99(38 \%)$ & $101(63 \%)$ & $200(47 \%)$ \\
\hline $1-4$ & $16(46 \%)$ & $85(52 \%)$ & $102(57 \%)$ & $20(43 \%)$ & $223(53 \%)$ & $163(62 \%)$ & $59(37 \%)$ & $222(53 \%)$ \\
\hline Total & 35 & 164 & 179 & 46 & 424 & 262 & 160 & 422 \\
\hline
\end{tabular}

\section{Dental fear and pain during extractions}

Children's self-assessment of dental fear resulted in $47 \%$ reporting no dental fear in conjunction with the extractions. During painful extractions, no fear was reported by $38 \%$, while in painless extractions, no fear was reported by $63 \%$ (Table 3). Dental fear was most common in the age group 11-15 years, followed by the age

group 7-11 years (Table 3).

\section{The child's cooperation while experiencing pain}

Full treatment acceptance was recorded in $73.6 \%$ during the extractions. In extractions reported to cause pain, $61 \%$ cooperated well (Table 4).

Table 4: Children's self-rated pain experiences and the degree of treatment acceptance, as rated by the dental personnel, on a scale 3-0, by Rud and Kisling.

\begin{tabular}{|c|c|c|c|}
\hline & Acceptance (3) & Indifferent Acceptance (2-0) & Total \\
\hline Pain & $135(61 \%)$ & $88(39 \%)$ & 223 \\
\hline No pain & $174(88 \%)$ & $23(12 \%)$ & 197 \\
\hline Total & 309 & 111 & 420 \\
\hline
\end{tabular}

The subjective assessment of pain=Yes or No was given by the child after the extraction (missing data $\mathrm{n}=16$ ).

\section{Treatment acceptance during parental presence}

Most of the patients (77\%) showed full treatment acceptance with parental presence in the dental room. The corresponding figure was 55\% for the parent not being present. All 37 three-yearold had their parents present in the dental room. Among the 166 seven-year-olds, two were not accompanied by an adult. Among the 11-year-olds, 26 out of 179 had no parent present. Among the 15-year-olds, 25 out of 45 managed on their own.

\section{Discussion}

This longitudinal, 5-year study voiced children's and adolescents' self-reported pain, discomfort, and fear during dental extractions. The main results showed that more than $60 \%$ of the dental extraction appointments involved painful experiences. Furthermore, pain was equally reported among the different age groups. The extracted teeth were predominantly performed in young schoolchildren. This may indicate that more vulnerable children were at risk experiencing pain at an early stage of their dental care encounters. Within the extraction procedure, the injection was most frequently reported responsible for the negative experiences. During decades, pain has been connected with dental extractions and concerned children and adolescents [17]. It could be reasoned that the injection techniques have not developed sufficiently, despite alternative anesthesia delivery methods, Computer controlled local anesthesia, Jet injectors, Iontophoresis and Computer controlled intraosseous anesthesia [18,19]. An explanation might

be that as these methods lie in the hands of numerous dentists, the injection performance may not be possible to calibrate. Other factors adding to the child's pain experience may be the dentists 
handling the onset of the anesthetic, the amount of delivered anesthesia, or a complementary anesthesia differently. A noteworthy result was that topical anesthesia was applied in only $35 \%$ by the dentists, which is astonishingly low given the scientific evidence in support of the technique, which is in alignment with earlier result [7]. Furthermore, adding to the children's uneasiness, discomfort was reported frequently during the extraction procedure. Thus, despite a complete anesthesia numbness, discomfort may have been experienced due to cracking sounds and/or the perceived pressure during the dental luxation. Discomfort has not been given much attention in the dental literature compared to general medical care [20]. The frequently reported discomfort also suggest that the dentists may not have practiced current theoretical knowledge sufficiently, which needs to be recognized $[7,8]$.

No studies among dentists have confirmed the Knowledge Transition to have improved regarding dental invasive procedures in general or oral anesthesia specifically $[21,22]$. An explanation may be the complex interaction between the applied injection technique and the dentist's way of psychological and pedagogical coaching [23-25]. Preferably, children should beforehand be prepared on anesthesia numbness sensation, luxation cracking sounds, increasing tissue-pressure during luxation, postoperative taste-sensation (iron, i.e., blood), and the subsequent inconvenience of not being able to enjoy a meal directly after the tooth extraction. Studies on Dental Fear and Anxiety (DFA) have showed that invasive dental procedures, such as extractions and injections, were by many children and adolescents associated with fear and anxiety [26,27]. In the current study, $47 \%$ of the extraction appointments were experienced without fear. From this perspective, the extractions were performed under satisfactory conditions. On the other hand, $53 \%$ of the children experienced fear, ranging from a bit nervous to terrified. Versloot et al. (2008) have reasoned that the level of dental anxiety may be of greater importance for the child's reaction than the injection technique itself [27]. Given that young patients' early pain encounters may not only trigger. Behavior Management Problems (BMP) and/or Dental Fear and Anxiety (DFA), but may also alter future pain responses, the results of this study are notable [28]. Contrary to the pain reports that were equally common in all age cohorts of the current study, DFA was more frequently reported in schoolchildren. This may define a vulnerable developmental stage, requiring parental presence and support. The relatively high proportion of extraction appointments performed with the parent does not present in the room calls for additional studies.

The current study presented that parental presence in the dental room was most frequent among children 3-12 years old, hereafter the presence significantly declined. Praxis has suggested that most parents are experts on their child's behavior and reactions. Perets and Zadick (1998) found that most parents would assist, should the dentist not succeed to manage their child [29]. Vasiliki (2016) also stated that children responded more positively in the dental room with parental presence [11]. Pfefferle et al. (1982) reasoned that this may be due to parents knowing their child's reactions and capabilities in challenging situations [30]. The current study demonstrated that most children cooperated fully during the dental extraction procedures despite experiencing discomfort or pain. A possible interpretation is that these children were not equipped to speak their needs, or they surrendered easily to authorities. Indirectly, the results also suggested that dentists practiced varied theoretical understanding, technical skills, and ethical attitudes during dental extraction procedures, not always in favor of the child. A limitation of this study may be argued that children below six years of age were not reliable to give accurate assessments of their experiences. However, there is a strong opinion among researchers that children from the age of 3-4 years are capable to communicate well and should be supported to express perceptions of discomfort and pain [31]. The strength of this study was that the results originated from a 5-year longitudinal perspective based on young patients' experiences from the extraction appointments. These results, regarding pain, discomfort and DFA, have not been contradicted in the recent literature and are valuable for clinical discussions. In conclusion, dental extractions continue to constitute one of the utmost intricate and challenging treatment situations for young patients. The oral injection was most frequently reported to cause pain, discomfort, and fear during dental extractions. Dentists should make efforts to prevent pain and discomfort, as well as utilize parental support for the child's sense of security during dental extractions.

\section{Acknowledgements}

This study was funded by the Public Dental Service, Region Västra Götaland and Region Örebro County, Sweden.

\section{Conflict of Interest}

The authors declare no conflicts of interest.

\section{References}

1 Krekmanova L, Bergius M, Robertson A, Sabel N, Hafstrom C (2009) Everyday- and dental-pain experiences in healthy Swedish 8-19year olds: an epidemiological study. Int J Paediatr Dent 6: 438-447.

2 Ghanei M, Arnrup K, Robertson A (2018) Procedural pain in routine dental care for children: a part of the Swedish BITA study. Eur Arch Paediatr Dent 19: 365-372.

3 Merdad L, El-Housseiny AA (2017) Do children's previous dental experience and fear affect their perceived oral health-related quality of life (OHRQoL)? BMC Oral Health 17: 47.

4 Klingberg G, Berggren U, Carlsson SG, Noren JG (1995) Child dental fear: cause-related factors and clinical effects. European Journal of Oral Sciences 6: 405-412.

5 Skaret E, Raadal M, Kvale G, Berg E (2000) Factors related to missed and cancelled dental appointments among adolescents in Norway. Eur J Oral Sci 108: 175-183. 
6 Monteiro J, Tanday A, Ashley PF, Parekh S, Alamri H (2020) Interventions for increasing acceptance of local anaesthetic in children and adolescents having dental treatment Cochrane Systematic Review Intervention Version published.

7 Wondimu B, Dahllof G (2005) Attitudes of Swedish dentists to pain and pain management during dental treatment of children and adoescents. Int J Paediatr Dent 15: 159-168.

8 Rasmussen JK, Frederiksen JA, Hallonsten AL, Poulsen S (2005) Danish dentists' knowledge, attitudes and management of procedural dental pain in children: association with demographic characteristics, structural factors, perceived stress during the administration of local analgesia and their tolerance towards pain. Paediatr Dent Int Journal15: 159-168.

9 https://www.thefreedictionary.com/discomfort

10 Ahuja S, Gandhi K, Malhotra R, Kapoor R, Maywad S (2018) Assessment of the effect of parental presence in dental operatory on the behavior of children aged 4-7 years. Indian Soc Pedod Prev Dent 36: 167-172.

11 Vasiliki B, Konstantinos A, Vassilis K, Nikolaos K, van Loveren C (2016) The effect of parental presence on the child's perception and cooperation during dental treatment. Eur Arch Paediatr Dent 17: 381-386.

12 Afshar H, Nakhjavani YB, Mahmoudi Gharaei J Paryab M, Zadhoosh S (2011) The Effect of Parental Presence on the 5-year-Old Children's Anxiety and Cooperative Behavior in the First and Second Dental Visit. Iran J Pediatr 21: 193-200.

13 (2005) Five Tips for Managing Pediatric Dental Anxiety.

14 Chapman HR, Kirby-Turner N (2005) Psychological Intrusion - An Overlooked Aspect of Dental Fear. Front Psychol. 2018; 9: 501.

15 Rules and rights. How dental services are regulated in Sweden.

16 Boka V, Arapostathis K, Charitoudis G, Veerkamp J, van Loveren C (2017) A study of parental presence/absence technique for child dental behaviour management. Eur Arch Paediatr Dent 18: 405-409.

17 Kühnisch J, Daubländer M, Klingberg G (2017) Best clinical practice guidance for local analgesia in paediatric dentistry: an EAPD policy document. Eur Arch Paediatr Dent 18(5): 313-321.

18 Zavattini A, Charalambous P (2018) Alternative practices of achieving anaesthesia for dental procedures: a review. J Dent Anesth Pain Med 18(2): 79-88.

19 Hachem C E, Kaloustian M K, Cerutti F (2019) Metallic syringe versus electronically assisted injection system: a comparative clinical study in children. Eur J Paediatr Dent 20: 320-324.
20 Mira S Staphorst, Marc A Benninga, Margriet Bisschoff, Irma Bon, Jan J V Busschbach, et al. (2017) The child's perspective on discomfort during medical research procedures: a descriptive study. BMJ Open 7(7): e016077.

21 Berlin H, List T, Ridell K, Klingberg G (2018) Dentists' attitudes towards acute pharmacological pain management in children and adolescents. Int J Paediatr Dent 2: 152-160.

22 Versloot J, Veerkamp JSJ, Hoogstraten J (2008) Children's self-reported pain at the dentist. Pain 137: 389-394.

23 Rud K (1973) The influence of mental development on children's acceptence of dental treatment. Scandinavian Journal of Dental Research 5: 343-352.

24 Carrillo-Díaz M, Crego A, Armfield J, Romero M (2013) The moderating role of dental expectancies on the relationship between cognitive vulnerability and dental fear in children and adolescents. Community Dent Oral Epidemiol 41: 269-278.

25 Cademartori MGP, Martins AR, Goettems RML (2017) Behavioral changes during dental appointments in children having tooth extractions. J Indian Soc Pedod Prev Dent 35(3): 223-228.

26 Jalevik B, Klingberg G (2002) Dental treatment, dental fear and behaviour management problems in children with severe enamel hypomineralization of their permanent first molars. Int J Paediatr Dent 12(1): 24-32.

27 Versloot J, Veerkamp JS, Hoogstraten J (2008) Pain behaviour and distress in children during two sequential dental visits: comparing a computerised anaesthesia delivery system and a traditional syringe. $\mathrm{Br}$ Dent J 1: 30-31.

28 You DS, Meagher MW (2016) Childhood Adversity and Pain Sensitization. Psychosom Med 9: 1084-1093.

29 Peretz B, Zadik D (1998) Attitudes of parents towards their presence in the operatory during dental treatments to their children. J Clin Pediatr Dent 23(1): 27-30.

30 Pfefferle JC, Fields HW, Posnick WR (1982) Child behaviour in the dental setting relative to parental presence. Pediatric Dentistry 4: 311-316.

31 von Baeyer CL, Jaaniste T, Vo HLT, Brunsdon G (2017) Systematic Review of Self-Report Measures of Pain Intensity in 3- and 4-Year-Old Children: Bridging a Period of Rapid Cognitive Development. The Journal of Pain 18(9): 1017-1026.
This work is licensed under Creative Commons Attribution 4.0 License

To Submit Your Article Click Here: Submit Article

DOI: $10.32474 /$ IPDOAJ.2020.05.000202

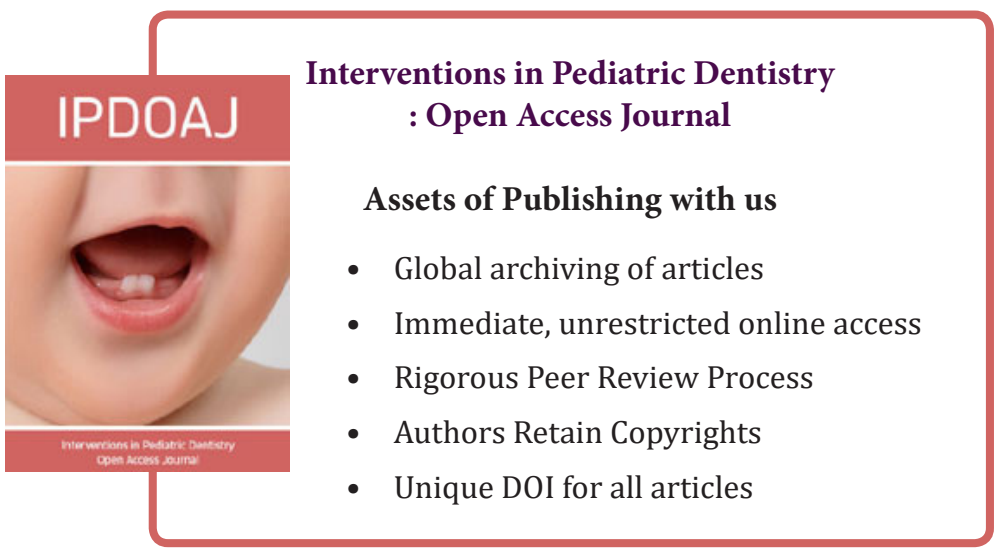

